\title{
Statistical approximation to Chlodowsky type $q$-Bernstein-Schurer-Stancu-Kantorovich operators
}

\author{
Behar Baxhaku* and Fevzi Berisha
}

(Communicated by Praveen AGARWAL)

\begin{abstract}
In this paper we introduce two kinds of Chlodowsky-type $q$-Bernstein-Schurer-Stancu- Kantorovich operators on the onbounded domain. The Korovkin type statistical approximation property of these operators are investigated. We investigated the rate of convergence for this approximation by means of the first and the second modulus of continuity. The rate of convergence is investigated by using Lipschitz classes of functions and the modulus of continuity of the derivative of the function. Then, we obtain point-wise estimate the Lipchitz type maximal function.
\end{abstract}

Keywords: Chlodowsky-type; q-Bernstein-Schurer-Stancu-Kantorivich; q-integer; modulus of continuity.

AMS Subject Classification (2010): Primary: 41A10 ; Secondary: 41A25; 41A36.

*Corresponding author

\section{Introduction}

The approximation theory is gaining importance, specifically the $q$-calculus due to its applications in various areas like Mathematics, Physics and Mechanics. Lupas [15] initiated the application of $q$-calculus in area of the approximation theory, and introduced the $q$-Bernstein polynomials. In 1997, it was Philips [18] who proposed and studied other $q$-Bernstein polynomials. Due to slow convergence of Bernstein polynomials some results on the expansion of $q$-Bernstein polynomials in orthogonal bases have been obtained in [4]. Proceeding further, several studies [5, 10,16,17], continued to obtain different properties of $q$-Bernstein polynomials and Durrmeyer variants. Approximations of vector values using $q$-Durrmeyer operators applied to random and fuzzy approximation are discussed in [9]. Agrawal et al. [2] introduced the Stancu type generalization of the Bernstein- Schurer operators. Other important operators are $q$-Bernstein types Integral Operators discussed in [5, 14].

Several studies investigated the $q$-analogue polynomials properties using statistical approximation. For instance in [19] $q$-Bernstein-Schurer; in [14] $q$-Bernstein-Schurer-Stancu-Kantorovich; [11] $q$-Baskakov-Kantorovich; in [1] $q$-Szász-King type operators are discussed and statistical approximation properties are demonstrated.

To motivate our paper, we take into consideration Karsli and Gupta [13] findings and their introduction of new operators to obtain local approximation properties and the rate of convergence. In theory of approximation, convergence is significant. This paper motive is to obtain a local approximation theorem and rate of convergence of Chlodowsky type $q$-Bernstein-Schurer-Stancu-Kantorovich operators and the weighted approximation properties. The results, turns out to have an order of approximation slightly better compared to the classical ChlodowskyBernstein-Stancu type operators in a certain subspace of continuous functions.

First, let us give some basic definitions and notations from $q$-calculus. Let $q>0$, for each nonnegative integer $k$,

Received : 01-November-2016, Accepted : 29-March-2017 
the $q$-integer $[k]_{q}$ and the $q$-factorial $[k]_{q}$ ! are defined by

$$
[k]_{q}=\left\{\begin{array}{lr}
\left(1-q^{k}\right) /(1-q), & q \neq 1 \\
k, & q=1,
\end{array}\right.
$$

and

$$
[k]_{q} != \begin{cases}{[k]_{q}[k-1]_{q} \ldots[1]_{q},} & k \geq 1 \\ 1, & k=1\end{cases}
$$

respectively. Then for $q>0$ and integers $n, k ; n \geq k \geq 0$, we have:

$$
[k+1]_{q}=1+q[k]_{q}, \quad \text { and } \quad[k]_{q}+q^{k}[n-k]=[n]_{q} .
$$

For integers $n, k ; n \geq k \geq 0$, the $q$-binomial coeficients is defined by

$$
\left[\begin{array}{l}
n \\
k
\end{array}\right]_{q}=\frac{[n]_{q} !}{[k]_{q} ![n-k]_{q} !}
$$

For an arbitrary function $f(x), q$-differential is given by:

$$
d_{q} f(x)=f(q x)-f(x) .
$$

The $q$-Jackson integral in the interval $[0, b]$ is defined as:

$$
\int_{0}^{b} f(t) d_{q} t=(1-q) b \sum_{j=0}^{\infty} f\left(q^{j} b\right) q^{j}, 0<q<1 .
$$

provided that sums converge absolutely. Suppose $0<a<b$. The $q$-Jackson integral in a generic interval $[a, b]$ is defined as

$$
\int_{a}^{b} f(t) d_{q} t=\int_{0}^{b} f(t) d_{q} t-\int_{0}^{a} f(t) d_{q} t, \quad 0<q<1 .
$$

More details regarding $q$-calculus can be found in [12].

Qiu Lin in [14] introduced the $q$-Bernstein-Schurer-Stancu-Kontorovich operators $K_{n, p}^{\alpha, \beta}(f ; q ; x): C[0,1+p] \rightarrow C[0,1]$, as follows

$$
K_{n, p}^{(\alpha, \beta)}(f ; q ; x)=\sum_{k=0}^{n+p}\left[\begin{array}{c}
n+p \\
k
\end{array}\right] x^{k} \cdot \prod_{s=0}^{n+p-k-1}\left(1-q^{s} x\right) \int_{0}^{1} f\left(\frac{t}{[n+1+\beta]_{q}}+\frac{q[k+\alpha]_{q}}{[n+1+\beta]_{q}}\right) d_{q} t
$$

$0 \leq x \leq 1$, for any $n \in N$ and $p \in N_{0}$ and $0 \leq \alpha \leq \beta$. H. Karsli and V. Gupta [13] introduced the $q$-Bernstein Chlodowsky polynomials as follow:

$$
C_{n}(f ; q ; x)=\sum_{k=0}^{n+p} f\left(\frac{[k]_{q}}{[n]_{q}} b_{n}\right)\left[\begin{array}{c}
n+p \\
k
\end{array}\right]\left(\frac{x}{b_{n}}\right)^{k} \prod_{s=0}^{n+p-k-1}\left(1-q^{s} \frac{x}{b_{n}}\right), \quad 0 \leq x \leq b_{n},
$$

where $b_{n}$ is a positive increasing sequences with the property $\lim _{n \rightarrow \infty} b_{n}=\infty, \lim _{n \rightarrow \infty} \frac{b_{n}}{[n]_{q}}=0$.

Recently, the Chlodowsky type $q$-Bernstein-Stancu-Kantorovich operators introduced by authors in [20] as

$$
K_{n, p}^{(\alpha, \beta)}(f ; q ; x)=\sum_{k=0}^{n+p}\left[\begin{array}{c}
n+p \\
k
\end{array}\right]\left(\frac{x}{b_{n}}\right)^{k} \prod_{s=0}^{n+p-k-1}\left(1-q^{s} \frac{x}{b_{n}}\right) \cdot \int_{0}^{1} f\left(\frac{\left(1+(q-1)[k]_{q}\right) t+[k]_{q}+\alpha}{[n+1]_{q}+\beta} b_{n}\right) d_{q} t
$$

where $n \in N$ and $p \in N_{0}$ and $\alpha, \beta \in N$ with $0 \leq \alpha \leq \beta, 0 \leq x \leq b_{n}, 0<q<1$.

This paper is organized as follows. In Section 2, we introduce the Chlodowsky type $q$-Bernstein-SchurerStancu-Kantorovich operators and evaluate the moments of these operators. In section 3 we obtain the statistical approximation properties of Korovkin type. In Section 4 we obtain the rate of convergence of the approximation, given in the previous section, by means of modulus of continuity of the function, elements of Lipschitz classes and the modulus of continuity of the derivative of the function. In Section 5 we obtain results of Local Approximation 


\section{Construction of the operators}

Let $b_{n}$ be increasing sequence of positive real number and let them satisfy the properties: $\lim _{n \rightarrow \infty} b_{n}=\infty$ that the sequence $\frac{b_{n}}{[n]_{q_{n}}}$ decrease to zero as $n \rightarrow \infty$, where $\left\{q_{n}\right\}$ is a sequence of real numbers such that $0<q_{n} \leq 1$ for all $n$ and $\lim _{n \rightarrow \infty} q_{n}=1$. We can introduce the Chlodowski-type $q$-Bernstein-Schures-Stancu-Kantorovich operators for a function $f$ as follows:

$$
T_{n, p}^{(\alpha, \beta)}(f ; q, x)=\sum_{k=0}^{n+p} p_{n+p, k}(q ; x) \int_{0}^{1} f\left(\frac{t \cdot b_{n}}{[n+1+\beta]_{q}}+\frac{q \cdot[k+\alpha]_{q} \cdot b_{n}}{[n+1+\beta]_{q}}\right) d_{q} t
$$

where $p_{n+p, k}(q ; x)=\left[\begin{array}{c}n+p \\ k\end{array}\right] \cdot\left(\frac{x}{b_{n}}\right)^{k} \cdot \prod_{s=0}^{n+p-k-1}\left(1-q^{s} \frac{x}{b_{n}}\right), 0 \leq x \leq b_{n}, n \in N$, and $p \in N_{0}$ and $\alpha, \beta$ a real parameters satisfying the conditions $0 \leq \alpha \leq \beta$. Clearly, $T_{n, p}^{(\alpha, \beta)}(f ; q, x)$ are positive linear operators.

To evaluate the main results, first let's formulate and proof some lemma's as follows:

Lemma 2.1. The moments of operators $T_{n, p}^{(\alpha, \beta)}(f ; q, x)$ are obtained for $f(t)=t^{i}, i=0,1,2$ we have:

i) $T_{n, p}^{(\alpha, \beta)}(1 ; q ; x)=1$,

ii) $T_{n, p}^{(\alpha, \beta)}(t ; q, x)=\frac{[n+p]_{q} q^{\alpha+1}}{[n+1+\beta]_{q}} x+\frac{b_{n}}{[n+1+\beta]_{q}} \cdot\left(\frac{1}{[2]_{q}}+q \cdot[\alpha]_{q}\right)$,

iii) $T_{n, p}^{(\alpha, \beta)}\left(t^{2} ; q, x\right)=\frac{q^{2 \alpha+3}[n+p]_{q} \cdot[n+p-1]_{q}}{[n+1+\beta]_{q}^{2}} \cdot x^{2}+\frac{[n+p]_{q}}{[n+1+\beta]_{q}^{2}} b_{n}$.

$\left.\cdot\left[\frac{2}{[2]_{q}} q^{\alpha+1}+q^{\alpha+2} \cdot\left(2 \cdot[\alpha]_{q}+q^{\alpha}\right)\right)\right] x+\frac{b_{n}^{2}}{[n+1+\beta]_{q}^{2}}\left(\frac{1}{[3]_{q}}+2 \frac{q \cdot[\alpha]_{q}}{[2]_{q}}+q^{2} \cdot[\alpha]_{q}^{2}\right)$.

Proof. It is obvious that: $\int_{0}^{1} 1 \cdot d_{q} t=1, \int_{0}^{1} t \cdot d_{q} t=\frac{1}{[2]_{q}}, \int_{0}^{1} t^{2} \cdot d_{q} t=\frac{1}{[3]_{q}}$.

i) Let's take into consideration the Binomial identity $(1-x)^{n+p-k}=\prod_{s=0}^{n+p-k-1}\left(1-q^{s} x\right)$, then we have:

$$
T_{n, p}^{(\alpha, \beta)}(1 ; q, x)=\sum_{k=0}^{n+p} p_{n+p, k}(q ; x) \cdot \int_{0}^{1} d_{q} t=1
$$

ii) $T_{n, p}^{(\alpha, \beta)}(t ; q, x)=\sum_{k=0}^{n+p} p_{n+p, k}(q ; x) \cdot \int_{0}^{1}\left(\frac{t b_{n}}{[n+1+\beta]_{q}}+\frac{q \cdot[k+\alpha]_{q} \cdot b_{n}}{[n+1+\beta]_{q}}\right) d_{q} t=$

$$
\begin{aligned}
& =\sum_{k=0}^{n+p} p_{n+p, k}(q ; x) \cdot \frac{1}{[n+1+\beta]_{q}} \cdot \frac{1}{[2]_{q}} \cdot b_{n}+\sum_{k=0}^{n+p} p_{n+p, k}(q ; x) \cdot \frac{q[k+\alpha]_{q} b_{n}}{[n+1+\beta]_{q}}= \\
& =\frac{q^{\alpha+1}[n+p]_{q}}{[n+1+\beta]_{q}} x+\frac{b_{n}}{[n+1+\beta]_{q}} \cdot\left(\frac{1}{[2]_{q}}+q \cdot[\alpha]_{q}\right) .
\end{aligned}
$$

using the equality which completes the proof of $i$ ) 
iii) After the calculation we obtain:

$$
\begin{aligned}
T_{n, p}^{(\alpha, \beta)}\left(t^{2} ; q, x\right)= & \sum_{k=0}^{n+p} p_{n+p, k}(q ; x) \int_{0}^{1}\left(\frac{t b_{n}}{[n+1+\beta]_{q}}+\frac{q \cdot[k+\alpha]_{q} b_{n}}{[n+1+\beta]_{q}}\right)^{2} d_{q} t= \\
= & \frac{b_{n}^{2}}{[n+1+\beta]_{q}^{2}} \sum_{k=0}^{n+p} p_{n+p, k}(q ; x) \cdot\left(\frac{1}{[3]_{q}}+2 q \cdot \frac{[k+\alpha]_{q}}{[2]_{q}}+q^{2} \cdot[k+\alpha]_{q}^{2}\right)= \\
& =\frac{b_{n}^{2}}{[n+1+\beta]_{q}^{2}} \cdot \frac{1}{[3]_{q}}+\frac{2 b_{n}^{2} q}{[2]_{q} \cdot[n+1+\beta]_{q}^{2}} \cdot[\alpha]_{q}+\frac{2 b_{n}^{2} q}{[2]_{q} \cdot[n+1+\beta]_{q}^{2}} \cdot q^{\alpha} \cdot \frac{x}{b_{n}} . \\
& {[n+p]_{q}+\frac{b_{n}^{2} q^{2}}{[n+1+\beta]_{q}^{2}}\left[[\alpha]_{q}^{2}+2[\alpha]_{q} \cdot q^{\alpha}[n+p]_{q} \cdot \frac{x}{b_{n}}+q^{2 \alpha}[n+p]_{q} \cdot \frac{x}{b_{n}}+q^{2 \alpha+1} \cdot \frac{x}{b_{n}^{2}}\right.} \\
& {\left.[n+p]_{q} \cdot[n+p-1]_{q}\right]=q^{2 \alpha+3} \cdot \frac{[n+p]_{q} \cdot[n+p-1]_{q}}{[n+1+\beta]_{q}} x^{2}+\frac{[n+p]_{q}}{[n+1+\beta]_{q}^{2}} b_{n} . } \\
& {\left[\frac{2}{[2]_{q}} q^{\alpha+1}+q^{\alpha+2} \cdot\left(2 \cdot[\alpha]_{q}+q^{\alpha}\right)\right] x+\frac{b_{n}^{2}}{[n+1+\beta]_{q}^{2}}\left(\frac{1}{[3]_{q}}+\frac{2 q \cdot[\alpha]_{q}}{[2]_{q}}+q^{2}[\alpha]_{q}^{2}\right) . }
\end{aligned}
$$

Lemma 2.2. For the operators $T_{n, p}^{(\alpha, \beta)}$ defined by (2.1), we have

$$
\sup _{0 \leq x \leq b_{n}} T_{n, p}^{(\alpha, \beta)}\left((t-x)^{2} ; q ; x\right) \leq b_{n}^{2}\left\{\left(\frac{[n+p]_{q}}{[n+1+\beta]_{q}} q q^{\alpha+1}-1\right)+\frac{2 \cdot(1+\alpha)[n+p]_{q}}{[n+1+\beta]_{q}}+\frac{(1+\alpha)^{2}}{[n+1+\beta]_{q}^{2}}\right\} .
$$

Proof. Using Lemma 2.1 and the linearity of the operators, we have:

$$
\begin{aligned}
T_{n, p}^{(\alpha, \beta)}\left((t-x)^{2}\right) & =T_{n, p}^{(\alpha, \beta)}\left(t^{2} ; q, x\right)-2 x T_{n, p}^{(\alpha, \beta)}(t ; q ; x)+x^{2} T_{n, p}^{(\alpha, \beta)}(1 ; q ; x)= \\
& =\left(q^{2 \alpha+3} \cdot \frac{[n+p]_{q} \cdot[n+p-1]_{q}}{[n+1+\beta]_{q}^{2}}-2 \cdot \frac{q^{\alpha+1} \cdot[n+p]_{q}}{[n+1+\beta]_{q}}+1\right) x^{2}+ \\
& +\left[\frac{[n+p]_{q}}{[n+1+\beta]_{q}^{2}} b_{n} \cdot\left(\frac{2}{[2]_{q}} q^{\alpha+1}+q^{\alpha+2} \cdot\left(2[\alpha]_{q}+q^{\alpha}\right)\right)-\right. \\
& \left.-2 \cdot \frac{b_{n}}{[n+1+\beta]_{q}}\left(\frac{1}{[2]_{q}}+q \cdot[\alpha]_{q}\right)\right] x+\frac{b_{n}^{2}}{[n+1+\beta]_{q}^{2}}\left(\frac{1}{[3]_{q}}+\frac{2 q \cdot[\alpha]_{q}}{[2]_{q}}+q^{2} \cdot[\alpha]_{q}^{2}\right)
\end{aligned}
$$

if we take the supremum on $\left[0, b_{n}\right]$, and considering $[n+p]_{q} \cdot[n+p-1]_{q} \cdot q \leq[n+p]_{q}^{2}$, we have:

$$
\left.T_{n, p}^{(\alpha, \beta)}\left((t-x)^{2}\right) ; q ; x\right) \leq b_{n}^{2}\left\{\left(\frac{[n+p]_{q}}{[n+1+\beta]_{q}} \cdot q^{\alpha+1}-1\right)^{2}+\frac{2 \cdot(1+\alpha)[n+p]_{q}}{[n+1+\beta]_{q}^{2}}+\frac{(1+\alpha)^{2}}{[n+1+\beta]_{q}^{2}}\right\} .
$$

We now redefine $T_{n, p}^{(\alpha, \beta)}(f ; q ; x)$ as follows:

$$
\tilde{T}_{n, p}^{(\alpha, \beta)}(f ; q, x)=\sum_{k=0}^{n+p} p_{n+p, k}(q ; x) \int_{0}^{1} f\left(\frac{t b_{n}}{[n+1]_{q}+\beta}+\frac{q[k]_{q}+\alpha}{[n+1]_{q}+\beta} b_{n}\right) d_{q} t .
$$

For the redefined operators, let us give the following Lemma.

Lemma 2.3. For $\tilde{T}_{n, p}^{(\alpha, \beta)}\left(t^{i} ; q, x\right), i=0,1,2$, one has

$$
\begin{aligned}
& \text { i) } \tilde{T}_{n, p}^{(\alpha, \beta)}(1 ; q, x)=1 \\
& \text { ii) } \tilde{T}_{n, p}^{(\alpha, \beta)}(t ; q, x)=\frac{[n+p]_{q}}{[n+1]_{+}+\beta} \cdot q x+\frac{b_{n}}{[n+1]_{q}+\beta}\left(\frac{1}{[2]_{q}}+q \alpha\right), \\
& \text { iii) } \tilde{T}_{n, p}^{(\alpha, \beta)}\left(t^{2} ; q, x\right)=\frac{[n+p]_{q}[n+p-1]_{q}}{\left([n+1]_{q}+\beta\right)^{2}} \cdot q^{3} x^{2}+\frac{[n+p]_{q} b_{n}}{\left([n+1]_{q}+\beta\right)^{2}}\left[\frac{2 q}{[2]_{q}}+q^{2}(2 \alpha+1)\right] x+ \\
& +\frac{b_{n}^{2}}{\left([n+1]_{q}+\beta\right)^{2}}\left[\frac{1}{[3]_{q}}+\frac{2 q \alpha}{[2]_{q}}+\alpha^{2} q^{2}\right] .
\end{aligned}
$$

Proof. 
i) It is obvious that $\tilde{T}_{n, p}^{(\alpha, \beta)}(1 ; q, x)=1$

ii) $\tilde{T}_{n, p}^{(\alpha, \beta)}(t ; q ; x)=\sum_{k=0}^{n+p} p_{n+p, k}(q ; x) \cdot \int_{0}^{1}\left(\frac{t b_{n}}{[n+1]_{q}+\beta}+\frac{q\left([k]_{q}+\alpha\right) b_{n}}{[n+1]_{q}+\beta}\right) d_{q} t=$

$$
\begin{aligned}
& =\frac{1}{[2]_{q}} \frac{b_{n}}{[n+1]_{q}+\beta}+\frac{q b_{n}}{[n+1]_{q}+\beta} \sum_{k=0}^{n+p} \frac{[n+p]_{q}[n+p-1]_{q} ![k]_{q}+\alpha}{[k]_{q} ![n+p-k]_{q} !} p_{n+p, k}(q ; x)= \\
& =\frac{1}{[2]_{q}} \frac{b_{n}}{[n+1]_{q}+\beta}+\frac{q[n+p]_{q}}{[n+1]_{q}+\beta} \frac{x b_{n}}{b_{n}}+\frac{q \alpha b_{n}}{[n+1]_{q}+\beta}=\frac{[n+p]_{q}}{[n+1]_{q}+\beta} q x+\frac{b_{n}}{[n+1]_{q}+\beta}\left(\frac{1}{[2]_{q}}+q \alpha\right)
\end{aligned}
$$

iii) $\tilde{T}_{n, p}^{(\alpha, \beta)}\left(t^{2} ; q ; x\right)=\sum_{k=0}^{n+p} p_{n+p, k}(q ; x) \cdot \int_{0}^{1}\left(\frac{t b_{n}}{[n+1]_{q}+\beta}+\frac{q\left([k]_{q}+\alpha\right) b_{n}}{[n+1]_{q}+\beta}\right)^{2} d_{q} t=$

$$
\begin{aligned}
& =\frac{b_{n}^{2}}{[n+1]_{q}+\beta}\left[\frac{1}{[3]_{q}}+\frac{2 q \alpha}{[2]_{q}}+\alpha^{2} q^{2}\right]+\frac{2 b_{n}^{2}[n+p]_{q} q \frac{x}{b_{n}}}{\left([n+1]_{q}+\beta\right)^{2}}\left(\frac{1}{[2]_{q}}+\alpha q\right)+\frac{q^{3} b_{n}^{2}[n+p]_{q}[n+p+1]_{q}}{\left([n+1]_{q}+s\right)^{2}} \frac{x^{2}}{b_{n}^{2}}= \\
& =\frac{[n+p]_{q}[n+p-1]_{q}}{\left([n+1]_{q}+\beta\right)^{2}} q^{3} x^{2}+\frac{[n+p]_{q} b_{n}}{\left([n+1]_{q}+\beta\right)^{2}}\left[\frac{2 q}{[2]_{q}}+q^{2}(2 \alpha+1)\right] x+\frac{b_{n}^{2}}{\left([n+1]_{q}+\beta\right)^{2}}\left[\frac{1}{[3]_{q}}+\frac{2 q \alpha}{[2]_{q}}+\alpha^{2} q^{2}\right]
\end{aligned}
$$

Lemma 2.4. For the operators $\tilde{T}_{n, p}^{(\alpha, \beta)}$ we have:

$$
\sup _{0 \leq x \leq b_{n}} \tilde{T}_{n, p}^{(\alpha, \beta)}\left((t-x)^{2} ; q ; x\right) \leq\left(\frac{[n+p]_{q}}{[n+1]_{q}+\beta}-1\right)^{2} b_{n}^{2}+\frac{2(1+\alpha)[n+p]_{q} b_{n}^{2}}{\left([n+1]_{q}+\beta\right)}+\frac{(1+\alpha)^{2} b_{n}^{2}}{\left([n+1]_{q}+\beta\right)}
$$

Proof. Based on linearity and positivity of operators and the Lemma 2.3, we have:

$$
\begin{aligned}
\tilde{T}_{n, p}^{(\alpha, \beta)}\left((t-x)^{2} ; q ; x\right) & =\tilde{T}_{n, p}^{(\alpha, \beta)}\left(t^{2} ; q ; x\right)-2 x \tilde{T}_{n, p}^{(\alpha, \beta)}(t ; x)+x^{2} \tilde{T}_{n, p}^{(\alpha, \beta)}(1 ; q ; x)= \\
= & x^{2}\left(\frac{[n+p]_{q}[n+p-1]_{q}}{\left([n+1]_{q}+\beta\right)^{2}}-2 \frac{[n+p]_{q}}{\left([n+1]_{q}+\beta\right)} q+1\right)+x\left(\frac{[n+p] b_{n}}{\left([n+1]_{q}+\beta\right)^{2}}\right. \\
& \left.\left(\frac{2 q}{[2]_{q}}+q^{2}(2 \alpha)-\frac{2 b_{n}}{[n+1]_{q}+\beta}\left(\frac{1}{[2]_{q}}+q \alpha\right)\right)\right)+\frac{b_{n}^{2}}{\left([n+1]_{q}+\beta\right)^{2}}\left[\frac{1}{[3]_{q}}+\frac{2 q \alpha}{[2]_{q}}+\alpha^{2} q^{2}\right] .
\end{aligned}
$$

Then:

$$
\sup _{0 \leq x \leq b_{n}} \tilde{T}_{n, p}^{(\alpha, \beta)}\left((t-x)^{2} ; q ; x\right) \leq\left(\frac{[n+p]_{q}}{[n+1]_{q}+\beta}-1\right)^{2} b_{n}^{2}+\frac{2(1+\alpha)[n+p]_{q} b_{n}^{2}}{\left([n+1]_{q}+\beta\right)}+\frac{(1+\alpha)^{2} b_{n}^{2}}{\left([n+1]_{q}+\beta\right)}
$$

\section{Statistical approximation of Korovkin type}

First, we introduce some notation and the basic definitions used in this paper. Let $A=\left(a_{j n}\right)$ be a non-negative infinite summability matrix. For a given sequence $x:=\left(x_{n}\right)$ the A-transform of $x$, denoted by $A x:=\left((A x)_{j}\right)$, is given by $(A x)_{j}=\sum_{n=1}^{\infty} a_{j n} x_{n}$, provided the series converges for each $\mathrm{j}$. A is said to be $\operatorname{regular}$ if $\lim _{j}(A x)_{j}=L$ whenever $\lim _{j}(x)_{j}=L$. Then $x=(x)_{j}$ is said to be A-statistically convergent to L i.e. $s t_{A}-\lim _{j}(x)_{j}=L$ if for every $\varepsilon>0, \lim _{j} \sum_{k:\left|x_{k}-L\right| \geq \varepsilon} a_{j k}=0[7,8]$. Replacing A by C1 then A is a Cesaro matrix of order one and A-statistical convergence is reduced to the statistical convergence. For $A=I$, the identity matrix then A-statistical convergence is called ordinary convergence.

Let $R$ denote the set of real numbers. A real function $\rho$ is called a weight function if it is continuous on $R$ and $\lim _{|x| \rightarrow \infty} \rho(x)=\infty, \rho(x) \geq 1$ for all $x \in R$.

Let us denote by $B_{\rho}(R)$ the weighted space of real-valued functions $f$ defined on $R$ with the property $|f(x)| \leq$ $M_{f} \rho(x)$ for all $x \in R$, where $M_{f}$ is a constant depending on the function $f$. We also consider the weighted subspace $C_{\rho}(\rho)$ of $B_{\rho}(R)$ given by

$$
C_{\rho}(R)=\left\{f \in B_{\rho}(R): f \text { continuous on } R\right\}
$$

endowed with the norm $\|\cdot\|_{\rho}$ where $\|f\|_{\rho}=\sup _{x \in R} \frac{|f(x)|}{\rho(x)}, B_{\rho}(R)$ and $C_{\rho}(R)$ are Banach space. 
The concept of statistical convergence in approximation theory by linear positive operators was used by Gadijev and Orhan [8]. They proved the Bohman-Korovkin-type approximation theorem for statistical convergence.

Theorem 3.1. (see [8]). If the sequence of linear positive operators $A_{n}: C[a, b] \rightarrow C[a, b]$ satisfies the conditions:

for $e_{i}(t)=t^{i}, i=0,1,2$, then for any $f \in C[a, b]$,

$$
s t_{A}-\lim _{n}\left\|A_{n}\left(e_{i} ; \cdot\right)-e_{i}\right\|_{C[a, b]}=0
$$

$$
s t_{A}-\lim _{n}\left\|A_{n}(f ; \cdot)-f\right\|_{C[a, b]}=0 .
$$

Using $A$-statistical convergence Duman and Orhan proved the following Bohman-Korovkin-type theorem ([6], theorem 3.1).

Theorem 3.2. Let $A=\left(a_{j n}\right)_{j, n}$ be a non negative regular summability matrix and let $\left(L_{n}\right)_{n}$ be sequences of positive linear operators from $C_{\rho_{1}}(R)$ into $B_{\rho_{2}} R$, where $\rho_{1}$ and $\rho_{2}$ satisfy:

$$
\lim _{|x| \rightarrow \infty} \frac{\rho_{1}}{\rho_{2}}=0,
$$

Then $s t_{A}-\lim _{n}\left\|L_{n} F_{v}-F_{v}\right\|_{\rho_{1}}=0, v=0,1,2$. where $F_{v}(x)=\frac{x^{v} \rho_{1}(x)}{1+x^{2}}, v=0,1,2$. Moreover, taking $A$ the Cesáro matrix of the first order, Theorem 3.2 implies

Corollary 3.1. ([6]) For any sequences $\left(T_{n}\right)_{n \geq 1}$ of linear positive operators acting from $C_{\rho_{0}}\left(R^{+}\right)$into $C_{\rho_{\lambda}}\left(R^{+}\right), \lambda>0$ one has

if and only if

$$
s t_{A}-\lim _{n}\left\|T_{n} f-f\right\|_{\rho_{\lambda}}=0, f \in C_{\rho_{0}}\left(R^{+}\right) .
$$

$$
s t_{A}-\lim _{n}\left\|T_{n} e_{i}-e_{i}\right\|_{\rho_{0}}=0, i=0,1,2,
$$

where $\rho_{0}=1+x^{2}$.

Let $q=q_{n}, 0<q_{n}<1$, be sequences satisfying the following conditions:

$$
s t_{A}-\lim _{n} q_{n}=1, s t_{A}-\lim _{n} q_{n}^{n}=a(a<1), s t_{A}-\lim _{n} \frac{b_{n}}{[n]_{q}}=0 .
$$

Theorem 3.3. If $q=\left(q_{n}\right)$ satisfy condition (3.4), for each function $f \in C_{\rho_{0}}\left(R^{+}\right)$, one has:

$$
s t_{A}-\lim _{n}\left\|T_{n, p}^{(\alpha, \beta)}(f ; q ; x)-f\right\|_{\rho_{0}}=0 .
$$

Proof. For all $f \in C_{\rho_{0}}\left(R^{+}\right)$, the operator $T_{n, p}^{(\alpha, \beta)}(f ; q, x)$ is defined on $\left[0, b_{n}\right]$. We extend it on $R^{+}$in the classical manner. Let $\tilde{T}_{n, p}^{(\alpha, \beta)}$ be defined as follows

$$
\tilde{T}_{n, p}^{(\alpha, \beta)}(f, q, x)=\left\{\begin{array}{cc}
T_{n, p}^{(\alpha, \beta)}(f ; q ; x) & \text { for } \quad x \in I_{b_{n}} \\
f(x), & x \in R^{+} \backslash I_{a_{n}} .
\end{array}\right.
$$

For each $n \in N$ the norm $\left\|\tilde{T}_{n, p}^{(\alpha, \beta)}(f ; q ; x)-f\right\|_{\rho_{0}}$ coincides with the norms of the element $\left(T_{n, p}^{(\alpha, \beta)}(f ; q ; x)-f\right)$ in the space $B_{\rho_{\lambda}}\left[0, b_{n}\right]$ where $\rho_{\lambda}=1+x^{2+\lambda}$, for $\lambda \geq 0$. Applying Corollary 3.1 to operators $T_{n} \equiv \tilde{T}_{n, p}^{\alpha, \beta}$, the proof of Theorem will be finished. In this respect, it is sufficient to prove that under one hypothesis, the operators verify the conditions given in (3.3). From Theorem 3.1, it is enough to prove that $s t_{A}-\lim _{n}\left\|\tilde{T}_{n, p}^{(\alpha, \beta)}\left(e_{i} ; q, x\right)-e^{i}\right\|_{\rho_{0}}=0$ for $e_{i}=t^{i}$ for $i=0,1,2$. It is clear that

From Lemma 2.1 case ii) we have

$$
s t_{A}-\lim _{n}\left\|\tilde{T}_{n, p}^{(\alpha, \beta)}(1 ; q, x)-1\right\|_{\rho_{0}}=0 .
$$

$$
\begin{aligned}
\left\|\tilde{T}_{n, p}^{(\alpha, \beta)}(t ; q, x)-x\right\|_{\rho_{0}} & =\sup _{x \in[0, \infty)} \frac{\left.\mid \tilde{T}_{n}^{(\alpha, \beta)}\left(t ; q_{n}, x\right)-x\right)}{1+x^{2}} \leq \sup _{0 \leq x \leq b_{n}} \frac{\left|T_{n}^{(\alpha, \beta)}\left(t ; q_{n}, x\right)-x\right|}{1+x^{2}}= \\
& =\sup _{0 \leq x \leq b_{n}} \frac{1}{1+x^{2}}\left\{\left|\frac{q^{\alpha+1}[n+p]_{q}}{[n+1+\beta]_{q}}-1\right| x\right\}+\frac{b_{n}}{[n+1+\beta]_{q}}\left(\frac{1}{[2]_{q}}+q[\alpha]_{q}\right) \leq \\
& \leq\left|\frac{q^{\alpha+1}[n+p]_{q}}{[n+1+\beta]_{q}}-1\right| b_{n}+\frac{b_{n}}{[n+1+\beta]_{q}}\left(\frac{1}{[2]_{q}}+q[\alpha]_{q}\right) \leq \\
& \leq\left|\frac{q^{\alpha+1}[n+p]_{q}}{[n+1+\beta]_{q}}-1\right| b_{n}+\frac{b_{n}}{[n]_{q}}(1+\alpha) .
\end{aligned}
$$


We denote $\theta_{n}=\left(\frac{q^{\alpha+1}[n+p]_{q}}{[n+1+\beta]_{q}}-1\right) \cdot b_{n}$, and $\gamma_{n}=\frac{b_{n}}{[n]_{q}}(1+\alpha)$. From (5), we get $s t_{A}-\lim _{n} \theta_{n}=s t_{A}-\lim _{n} \gamma_{n}=0$.

Now, for a given $\epsilon>0$, let's define the following sets:

$$
\begin{aligned}
& T_{1}=\left\{n:\left\|T_{n, p}^{(\alpha, \beta)}(t ; q ; x)-x\right\|_{\rho_{0}} \geq \epsilon\right\}, \\
& T_{2}=\left\{n:\left(\frac{q^{\alpha+1}[n+p]_{q}}{[n+1+\beta]_{q}}-1\right) b_{n} \geq \epsilon / 2\right\}, \\
& T_{3}=\left\{n: \frac{(\alpha+1) b_{n}}{[n]_{q}} \geq \epsilon / 2\right\} .
\end{aligned}
$$

Then, from (3.5), we obtain $T_{1} \subseteq T_{2} \cup T_{3}$, so which implies that $\sum_{k \in T_{1}} a_{n k} \leq \sum_{k \in T_{1}} a_{n k}+\sum_{k \in T_{2}} a_{n k}$ and hence

$$
s t_{A}-\lim _{n} \| K_{n, p}^{(\alpha, \beta)}\left(t ; q_{n}, \cdot-x \|_{\rho_{0}}=0 .\right.
$$

From Lemma Lemma 2.1 case iii) we have

$$
\begin{aligned}
&\left\|\tilde{T}_{n, p}(\alpha, \beta)\left(t^{2} ; q ; x\right)-x^{2}\right\|_{\rho_{0}}=\sup _{x \in[0, \infty)} \frac{\left|\tilde{T}_{n}^{(\alpha, \beta)}\left(t^{2} ; q, x\right)-x^{2}\right|}{1+x^{2}} \leq \sup _{0 \leq x \leq b_{n}} \frac{\left|T_{n}^{(\alpha, \beta)}\left(t^{2} ; q, x\right)-x^{2}\right|}{1+x^{2}} \\
& \quad \leq \sup _{0 \leq x \leq b_{n}} \frac{1}{1+x^{2}}\left\{\left|\frac{q^{2 \alpha+3}[n+p]_{q}[n+p-1]_{q}}{[n+1+\beta]_{q}^{2}}-1\right| x^{2}+\frac{[n+p]_{q}}{[n+1+\beta]_{q}} b_{n} .\right. \\
&\left.\left(\frac{2}{[2]_{q}} q^{\alpha+1}+q^{\alpha+2}\left(2[\alpha]_{q}+q^{\alpha}\right)\right) x+\frac{b_{n}^{2}}{[n+1+\beta]_{q}^{2}}\left(\frac{1}{[3]_{q}}+\frac{2 q[\alpha]_{q}}{[2]_{q}}+q^{2}[\alpha]_{q}^{2}\right)\right\} \\
& \leq\left|\frac{q^{2 \alpha+2}[n+p]_{q}^{2}}{[n+1+\beta]_{q}^{2}}-1\right|+\frac{[n+p]_{q_{n}} q_{n}^{2 \alpha+2} b_{n}}{[n+1+\beta]_{q}^{2}}+\frac{(2+2 \alpha)[n+p]_{q_{n}} b_{n}}{[n+1+\beta]_{q_{n}}^{2}}+\frac{(1+\alpha)^{2} b_{n}^{2}}{[n+1+\beta]_{q_{n}}^{2}} \\
& \leq\left|\frac{[n+p]_{q_{n}}^{2} q^{2 \alpha+2}}{[n+1+\beta]_{q_{n}}^{2}}-1\right|+\frac{(3+2 \alpha) b_{n}}{[n]_{q_{n}}}\left(1+\frac{[p]_{q_{n}}}{[n]_{q_{n}}}\right)+\frac{(1+\alpha)^{2}}{[n]_{q_{n}}^{2}} b_{n}^{2}=\alpha_{n}+\beta_{n}+\gamma_{n},
\end{aligned}
$$

from (3.4) and $[n+p]_{q_{n}}=[n]_{q_{n}}+q_{n}^{n}[p]_{q_{n}},[n+1+\beta]_{q_{n}}=[n]_{q_{n}}+q_{n}^{n}[\beta+1]$, then we have $s t-\lim _{n} \alpha_{n}=s t-\lim \beta_{n}=$ st $-\lim _{n} \gamma_{n}=0$.

Let $\epsilon>0$. Then, we define the following sets:

$$
\begin{aligned}
& U=\left\{k:\left\|\tilde{T}_{n, p}^{(\alpha, \beta)}\left(t^{2} ; q ; x\right)-x^{2}\right\|_{\rho_{0}} \geq \epsilon\right\}, \\
& U_{1}=\left\{k: \alpha_{k} \geq \epsilon / 3\right\}, \\
& U_{2}=\left\{k: \beta_{k} \geq \epsilon / 3\right\}, \\
& U_{3}=\left\{k: \gamma_{k} \geq \epsilon / 3\right\} .
\end{aligned}
$$

Clearly, $U \subseteq U_{1} \cup U_{2} \cup U_{3}$, which implies that $\sum_{k \in U} a_{n k} \leq \sum_{k \in U_{1}} a_{n k}+\sum_{k \in U_{2}} a_{n k}+\sum_{k \in U_{3}} a_{n k}$. Thus,

$$
s t_{A}-\lim _{n \rightarrow \infty}\left\|\tilde{T}_{n, p}^{(\alpha, \beta)}\left(t^{2} ; q, x\right)-x^{2}\right\|_{\rho_{0}}=0 .
$$

Theorem 3.4. If $q=\left(q_{n}\right)$, satisfy (3.4), then $\forall f \in C_{\rho_{0}}\left(R^{+}\right)$,

$$
s t_{A}-\lim _{n}\left\|T_{n, p}^{(\alpha, \beta)}(f ; q ; x)-f\right\|_{\rho_{0}}=0
$$

Proof. Let $\tilde{T}_{n, p}^{(\alpha, \beta)}$ be defined as:

$$
\tilde{T}_{n, p}^{*(\alpha, \beta)}(f, q, x)=\left\{\begin{array}{cc}
\left(\tilde{T}_{n, p}^{(\alpha, \beta)} f\right)(x) & \text { for } \quad 0 \leq x \leq b_{n} \\
f(x), & x>b_{n} .
\end{array}\right.
$$

For each $n \in N$, the norm $\left\|\tilde{T}_{n, p}^{*(\alpha, \beta)}(f ; q ; x)-f\right\|_{\rho_{\lambda}}$ coincides with the norm of the element $\left(\tilde{T}_{n, p}^{*} f-f\right)$ in the space $B_{\rho_{\lambda}}\left[0, b_{n}\right]$, for any $\lambda \geq 0$.

Applying Corollary 3.1 to the operators $T_{n} \equiv \tilde{T}_{n, p}^{*(\alpha, \beta)}$, the proof of Theorem will be finished. In this respect, it is sufficient to prove that, under our hypotheses, the operators verify the conditions given at (3.3). From Theorem 3.1, it is enough to prove that $s t_{A}-\lim _{n}\left\|\tilde{T}_{n, p}^{(\alpha, \beta)}\left(e_{i} ; q, x\right)-e_{i}\right\|_{\rho_{0}}=0$ for $e_{i}=t^{i}, \mathrm{i}=0,1,2$. From Lemma 2.3 case (i), we can easily get

$$
s t_{A}-\lim _{n}\left\|\tilde{T}_{n, p}^{(\alpha, \beta)}(1 ; q, x)-1\right\|_{\rho_{0}}=0 .
$$


From Lemma 2.3 case (ii) we have

$$
\begin{aligned}
\| \tilde{T}_{n, p}^{(\alpha, \beta)}(t ; q, x)-x & \|_{\rho_{0}}=\sup _{x \in[0, \infty)} \frac{\left|\tilde{T}_{n, p}^{*(\alpha, \beta)}(t ; q, x)-x\right|}{1+x^{2}}=\sup _{0 \leq x \leq b_{n}} \frac{\left|\tilde{T}_{n, p}^{*(\alpha, \beta)}(t ; q, x)-x\right|}{1+x^{2}} \\
& \leq \sup _{0 \leq x \leq b_{n}} \frac{\left|\frac{[n+p]_{q} q}{[n+1]_{q}+\beta}-1\right| x+\frac{b_{n}}{[n+1]_{q}+\beta}\left(\frac{1}{[2]_{q}}+q[\alpha]_{q} \alpha\right)}{1+x^{2}} \leq\left|\frac{[n+p]_{q} q}{[n+1]_{q}+\beta}-1\right| b_{n} \\
& +\frac{b_{n}}{[n+1]_{q}+\beta}\left(\frac{1}{[2]_{q}}+q[\alpha]_{q}\right) \leq\left|\frac{[n+p]_{q} q_{n}}{[n+1]_{q}+\beta}-1\right| b_{n}+\frac{b_{n}}{[n]_{q_{n}}}(1+\alpha) .
\end{aligned}
$$

We denote $\theta_{n}=\left(\frac{[n+p]_{q} q}{[n+1]_{q}+\beta}-1\right) \cdot b_{n}$ and $\omega_{n}=\frac{b_{n}}{[n]_{q_{n}}}(1+\alpha)$.

From (3.4), we obtain $s t_{A}-\lim _{n} \theta_{n}=\lim _{n} \omega_{n}=0$, Now for a given $\epsilon>0$, let us define the following sets;

$$
\begin{aligned}
& \tilde{U}=\left\{k:\left\|\tilde{T}_{n, p}^{*(\alpha, \beta)}(t ; q ; x)-x\right\| \geq \epsilon\right\}, \\
& \tilde{U}_{1}=\left\{k:\left(\frac{q_{k}[k+p]_{q_{k}}}{[k+1]_{q_{k}}+\beta}-1\right) b_{k} \geq \epsilon / 2\right\}, \\
& \tilde{U}_{2}=\left\{k: \frac{(\alpha+1) b_{k}}{[k]_{q_{k}}} \geq \epsilon / 2\right\} .
\end{aligned}
$$

From (3.6), one can see that $\tilde{U} \subseteq \tilde{U}_{1} \cup \tilde{U}_{2}$, which implies that $\sum_{k \in \tilde{U}} a_{n k} \leq \sum_{k \in \tilde{U}_{1}} a_{n k}+\sum_{k \in \tilde{U}_{2}} a_{n k}$ and hence

Similarly,

$$
s t_{A}-\lim _{n}\left\|\tilde{T}_{n, p}^{*(\alpha, \beta)}(t ; q ; x)-x\right\|_{\rho_{0}}=0 .
$$

$$
\begin{aligned}
& \left\|\tilde{T}_{n, p}^{*(\alpha, \beta)}\left(t^{2} ; q ; x\right)-x^{2}\right\|_{\rho_{0}}=\sup _{0 \leq x<+\infty} \frac{\left|\tilde{T}_{n, p}^{*(\alpha, \beta)}\left(t^{2} ; q ; x\right)-x^{2}\right|}{1+x^{2}}=\sup _{0 \leq x \leq b_{n}} \frac{\left|T_{n, p}^{(\alpha, \beta)}\left(t^{2} ; q ; x\right)-x^{2}\right|}{1+x^{2}} \leq \\
& \sup _{0 \leq x \leq b_{n}} \frac{1}{1+x^{2}}\left\{\left|q^{3} \frac{[n+p]_{q}[n+p-1]_{q}}{\left([n+1]_{q}+\beta\right)^{2}}-1\right| x^{2}+\frac{[n+p]_{q} b_{n}}{\left([n+1]_{q}+\beta\right)^{2}}\left[\frac{2 q}{[2]_{q}}+q^{2}(2 \alpha+1)\right] x+\right. \\
& \left.\frac{b_{n}^{2}}{\left([n+1]_{q}+\beta\right)^{2}}\left[\frac{1}{[3]_{q}}+\frac{2 q \alpha}{[2]_{q}}+\alpha^{2} q^{2}\right]\right\} \leq\left|\left(\frac{[n+p]_{q} q}{[n+1]_{q}+\beta}\right)^{2}-1\right|+\frac{[n+p]_{q} b_{n}(3+2 \alpha)}{\left([n+1]_{q}+\beta\right)^{2}}+\frac{(1+\alpha)^{2} b_{n}^{2}}{[n]_{q_{n}}^{2}} \\
& =\tilde{\alpha_{n}}+\tilde{\beta_{n}}+\tilde{\gamma_{n}} .
\end{aligned}
$$

From (3.4) we obtain $s t-\lim _{n} \tilde{\alpha_{n}}=s t-\lim _{n} \tilde{\beta_{n}}=s t-\lim _{n} \tilde{\gamma_{n}}=0$.

Here for a given $\epsilon>0$, let us define the following sets:

$$
\begin{aligned}
& K=\left\{k:\left\|\tilde{T}_{n, p}^{*(\alpha, \beta)}\left(t^{2} ; q ; x\right)-x^{2}\right\|_{\rho_{0}} \geq 0\right\}, \\
& K_{1}=\left\{k: \alpha_{k} \geq \epsilon / 3\right\}, \\
& K_{2}=\left\{k: \beta_{k} \geq \epsilon / 3\right\}, \\
& K_{3}=\left\{k: \gamma_{k} \geq \epsilon / 3\right\} .
\end{aligned}
$$

Then we obtain $K \subseteq K_{1} \cup K_{2} \cup K_{3}$, which implies that $\sum_{k \in K} a_{n, k} \leq \sum_{k \in K_{1}} a_{n, k}+\sum_{k \in K_{2}} a_{n, k}+\sum_{k \in K_{3}} a_{n, k}$, and hence

$$
s t_{A}-\lim _{n}\left\|\tilde{T}_{n, p}^{*(\alpha, \beta)}\left(t^{2} ; q ; x\right)-x^{2}\right\|_{\rho_{0}}=0 .
$$

\section{Order of convergence}

Let $f \in C_{B}[0,+\infty)$ such that $\mathrm{f}$ is uniformly continuous for any $\delta>0$. The usual modulus of continuity for $f$ is defined as

$$
\omega(f ; \delta)=\sup _{\substack{|t-x| \leq \delta \\ t, x \in[0,+\infty)}}|f(t)-f(x)| .
$$

For $f \in C_{B}[0,+\infty)$ and any $t, x \in[0,+\infty)$, we have:

so for any $\delta>0$ we get:

$$
|f(t)-f(x)| \leq \omega(f ;|t-x|)
$$




$$
\omega(f,|t-x|) \leq\left\{\begin{array}{c}
\omega(f, \delta), \quad|t-x|<\delta \\
\omega\left(f, \frac{(t-x)^{2}}{\delta}\right), \quad|t-x| \geq \delta .
\end{array}\right.
$$

In the light of $\omega(f ; \lambda \delta) \leq(1+\lambda) \cdot \omega(f ; \delta)$ for $\lambda>0$, it is clear that we have:

for any $t, x \in[0,+\infty)$ and $\delta>0$.

$$
|f(t)-f(x)| \leq\left(1+\delta^{-2}(t-x)^{2}\right) \omega(f, \delta),
$$

Theorem 4.1. If $f \in C_{B}[0,+\infty)$, and $q=q_{n}$ be sequences such that $0<q_{n}<1$, we have:

where, $\delta_{n, q}(x)=T_{n, p}^{(\alpha, \beta)}\left((t-x)^{2}, q, x\right)$.

$$
\left|T_{n, p}^{(\alpha, \beta)}(f ; q ; x)-f(x)\right| \leq 2 \omega\left(f ; \sqrt{\delta_{n, p}(x)}\right),
$$

Proof. The fact that $T_{n, p}^{(\alpha, \beta)}(f ; q ; x)$ is positive linear operator, and applying the property of modulus continuity:

we have

$$
|f(t)-f(x)| \leq \omega(f, \delta)\left(\frac{|t-x|}{\delta}+1\right), \quad \text { for any } \delta>0,
$$

$$
\left|T_{n, p}^{(\alpha, \beta)}(f ; q ; x)-f(x)\right| \leq \mid T_{n, p}^{(\alpha, \beta)}(|f(t)-f(x)| ; q, x) \leq \omega(f ; \delta)\left(T_{n, p}^{(\alpha, \beta)}(1, q, x)+\frac{1}{\delta} T_{n, p}^{(\alpha, \beta)}(|t-x| ; q, x)\right) .
$$

From Hölder inequality, we know that: $\frac{1}{p}+\frac{1}{q}=1 ; q=2$, and $p=2$, we get:

$$
\left|T_{n, p}^{(\alpha, \beta)}(f ; q ; x)-f(x)\right| \leq \omega(f ; \delta)\left(T_{n, p}^{(\alpha, \beta)}(1)+\frac{1}{\delta}\left\{T_{n, p}^{(\alpha, \beta)}\left((t-x)^{2} ; q, x\right)\right)\right\}^{1 / 2} .
$$

Let's choose $\left.\delta=\delta_{n, q}=\left\{T_{n, p}^{(\alpha, \beta)}\left((t-x)^{2} ; q, x\right)\right)\right\}^{1 / 2}$, then we have:

$$
\left|T_{n, p}^{(\alpha, \beta)}(f ; q ; x)-f(x)\right| \leq 2 \omega\left(f ; \delta_{n, p}(x)\right) .
$$

Theorem 4.2. Let $\left(q_{n}\right)$ be sequence of real numbers such that $0<q_{n}<1$ and $\lim _{n \rightarrow \infty} q_{n}=1$. If $f^{\prime} \in C_{B}[0, \infty)$ and $\omega\left(f^{\prime} ; \delta\right)$ are modulus of continuity of $f^{\prime}(x)$ in $[0, C]$. Then,

$$
\begin{aligned}
& \left|T_{n, p}^{(\alpha, \beta)}(f ; q ; x)-f(x)\right| \leq M\left(C\left|u_{n}-1\right|+v_{n}\right)+2\left\{C^{2}\left(u_{n}-1\right)^{2}+C \frac{(\alpha+1)[n+p]_{q}}{[n+1+\beta]_{q}}+\frac{b_{n}^{2}(1+\alpha)^{2}}{[n+1+\beta]_{q}^{2}}\right\}^{1 / 2} \cdot \omega\left(f^{\prime}, \delta\right), \\
& \text { where } M \text { is a positive constant and } u_{n}=\frac{q^{\alpha+1}[n+p]_{q}}{[n+1+\beta]_{q}}, v_{n}=\frac{b_{n}}{[n+1+\beta]_{q}}\left(\frac{1}{[2]_{q}}+q[\alpha]_{q}\right) .
\end{aligned}
$$

Proof. Using Langrange's theorem we have:

$$
\begin{aligned}
f\left(\frac{t b_{n}}{[n+1+\beta]_{q}}+\frac{q[k+\alpha]_{q}}{[n+1+\beta]} b_{n}\right)-f(x)=\left(\frac{t b_{n}}{[n+1+\beta]_{q}}+\frac{q[k+\alpha]_{q} b_{n}}{[n+1+\beta]_{q}}-x\right) f^{\prime}(\theta)= \\
=\left(\frac{t b_{n}}{[n+1+\beta]_{q}}+\frac{q[k+\alpha]_{q} b_{n}}{[n+1+\beta]_{q}}-x\right) f^{\prime}(x)+\left(\frac{t b_{n}}{[n+1+\beta]_{q}}+\frac{q[k+\alpha]_{q} b_{n}}{[n+1+\beta]_{q}}-x\right)\left(f^{\prime}(\theta)-f(x)\right)
\end{aligned}
$$

where $x<\theta<\frac{t b_{n}}{[n+1+\beta]_{q}}+\frac{q[k+\alpha]_{q}}{[n+1+\beta]_{q}}$. Using the last equality, we can write the following inequality:

$$
T_{n, p}^{(\alpha, \beta)}(f ; q ; x)-f(x)=f^{\prime}(x) T_{n, p}^{(\alpha, \beta)}((t-x), q, x)+T_{n, p}^{(\alpha, \beta)}\left((t-x) \cdot\left(f^{\prime}(\theta)-f^{\prime}(x)\right) ; q, x\right) .
$$

Hence,

$$
\begin{gathered}
\left|T_{n, p}^{(\alpha, \beta)}(f ; q ; x)-f(x)\right| \leq\left|f^{\prime}(x)\right|\left|T_{n, p}^{(\alpha, \beta)}((t-x), q, x)\right|+T_{n, p}^{(\alpha, \beta)}\left(|t-x| \cdot\left|f^{\prime}(\theta)-f^{\prime}(x)\right| ; q, x\right) \\
\leq M\left(C\left|u_{n}-1\right|+v_{n}\right)+T_{n, p}^{(\alpha, \beta)}\left(|t-x| \cdot\left|f^{\prime}(\theta)-f^{\prime}(x)\right| ; q, x\right) .
\end{gathered}
$$

Applying the following well known property of modulus of continuity

we have

$$
|f(t)-f(x)| \leq \omega(f, \delta)\left(1+\frac{|t-x|}{\delta}\right), \text { for any } \delta>0,
$$

$$
\left|T_{n, p}^{(\alpha, \beta)}(f ; q ; x)-f(x)\right| \leq M\left(C\left|u_{n}-1\right|+v_{n}\right)+\omega\left(f^{\prime} ; \delta\right)\left[T_{n, p}^{(\alpha, \beta)}(|t-x| ; q, x)+\frac{1}{\delta} T_{n, p}^{(\alpha, \beta)}\left((t-x)^{2} ; q, x\right)\right] .
$$

Note: $|\theta-x| \leq\left|\frac{t b_{n}}{[n+1+\beta]_{q}}+\frac{q[k+\alpha]_{q}}{[n+1+\beta]_{q}} b_{n}-x\right|$.

Using the Cauchy-Schwarz inequality for the second term, then we have:

$$
\left|T_{n, p}^{(\alpha, \beta)}(f ; q ; x)-f(x)\right| \leq M\left(C\left|u_{n}-1\right|+v_{n}\right)+\omega\left(f^{\prime} ; \delta\right)\left\{T_{n, p}^{(\alpha, \beta)}\left((t-x)^{2} ; q, x\right\}^{1 / 2}+\frac{\omega\left(f^{\prime} ; \delta\right)}{\delta} T_{n, p}^{(\alpha, \beta)}\left((t-x)^{2} ; q, x\right) .\right.
$$


Now, if we get:

$$
\begin{aligned}
\sup _{0 \leq x \leq C} T_{n, p}^{(\alpha, \beta)}\left((t-x)^{2} ; q, x\right) & \leq \sup _{x \in[0, C]}\left\{x^{2}\left(u_{n}-1\right)^{2}+x \frac{(1+\alpha)[n+p]_{q}}{[n+1+\beta]} b_{n}+\frac{b_{n}^{2}(1+\alpha)^{2}}{[n+1+\beta]^{2}}\right\} \\
& \leq C^{2}\left(u_{n}-1\right)^{2}+C \frac{(1+\alpha)[n+p]_{q}}{[n+1+\beta]_{q}} b_{n}+\frac{b_{n}^{2}(1+\alpha)^{2}}{[n+1+\beta]_{q}^{2}} .
\end{aligned}
$$

Thus,

$$
\begin{aligned}
\left|T_{n, p}^{(\alpha, \beta)}(f ; q, x)-f(x)\right| & \leq M\left[C\left|u_{n}-1\right|+v_{n}\right]+\omega\left(f^{\prime}, \delta\right)\left[C^{2}\left(u_{n}-1\right)^{2}+C \frac{(1+\alpha)[n+p]_{q}}{[n+1+\beta]_{q}^{2}} b_{n}+\frac{b_{n}^{2}}{[n+1+\beta]_{q}^{2}}(1+\alpha)^{2}\right]^{1 / 2} \\
& +\frac{\omega\left(f^{\prime}, \delta\right)}{\delta}\left\{C^{2}\left(u_{n}-1\right)^{2}+C \frac{(1+\alpha)[n+p]_{q}}{[n+1+\beta]_{q}^{2}} b_{n}+\frac{b_{n}^{2}(1+\alpha)^{2}}{[n+1+\beta]_{q}^{2}}\right\} .
\end{aligned}
$$

Let's write: $\delta=\left\{C^{2}\left(u_{n}-1\right)^{2}+C \frac{(1+\alpha)[n+p]_{q}}{[n+1+\beta]_{q}^{2}} b_{n}+\frac{b_{n}^{2}(1+\alpha)^{2}}{[n+1+\beta]_{q}^{2}}\right\}^{1 / 2}$, then we have:

$$
\left|T_{n, p}^{(\alpha, \beta)}(f, q, x)-f(x)\right| \leq M\left(C\left|u_{n}-1\right|+v_{n}\right)+2\left\{C^{2}\left(u_{n}-1\right)^{2}+C \frac{(1+\alpha)[n+p]_{q}}{[n+1+\beta]_{q}}+\frac{b_{n}^{2}(1+\alpha)^{2}}{[n+1+\beta]_{q}^{2}}\right\}^{1 / 2} \cdot \omega\left(f^{\prime}, \delta\right) .
$$

Lemma 4.1. Let $\left(q_{n}\right)$ be sequences of real numbers such that $0<q_{n}<1, \lim _{n \rightarrow \infty} q_{n}=1$, if $f \in \operatorname{Lip}_{K}(\gamma)$ and $x \in[0, C], C>$ 0 . Then we have,

$$
\left.\| T_{n, p}^{(\alpha, \beta)}(f, q, x)-f\right) \|_{C\left[0, b_{n}\right]} \leq K\left\{C T_{n, p}^{(\alpha, \beta)}\left((t-x)^{2} ; q, x\right)\right\}^{\alpha / 2}
$$

Proof. By the linearity and monotonicity of the operators $T_{n, p}^{(\alpha, \beta)}(f, q, x)$, we have,

$$
\left.\left|T_{n, p}^{(\alpha, \beta)}(f, q, x)-f(x)\right| \leq T_{n, p}^{(\alpha, \beta)}|f(t)-f(x)| ; q, x\right) \leq K \cdot T_{n, p}^{(\alpha, \beta)}\left(|t-x|^{\gamma} ; q, x\right) .
$$

Using Holder inequality with $p_{1}=\frac{2}{\gamma}$, and $p_{2}=\frac{2}{2-\gamma}$, then $\frac{1}{p_{1}}+\frac{1}{p_{2}}=1$. We can write:

This implicates

$$
\left|T_{n, p}^{(\alpha, \beta)}(f, q, x)-f(x)\right| \leq K \cdot\left(C T_{n, p}^{(\alpha, \beta)}(t-x)^{2} ; q, x\right)^{\gamma / 2} \cdot T_{n, p}^{(\alpha, \beta)}(1, q, x)^{\frac{2-\gamma}{2}} .
$$

$$
\left.\| T_{n, p}^{(\alpha, \beta)}(f, q, x)-f\right) \|_{C\left[0, b_{n}\right]} \leq K\left\{C T_{n, p}^{(\alpha, \beta)}\left((t-x)^{2} ; q, x\right)\right\}^{\alpha / 2} .
$$

Theorem 4.3. Let $0<a \leq 1$ and $E$ by any subset of the interval $[0,+\infty)$. Then, if $f \in C_{B}[0,+\infty)$ is locally Lip $(a)$, i.e the condition

$$
|f(t)-f(x)| \leq L|t-x|^{a}, \quad t \in E \quad \text { and } \quad x \in[0,+\infty),
$$

holds, then, for each $x \in[0,+\infty)$, we have:

$$
\left|T_{n, p}^{\alpha, \beta}(f, q, x)-f(x)\right| \leq L\left\{T_{n, p}^{\alpha, \beta}\left((t-x)^{2}, q, x\right)+2 d^{a}(X, E)\right\}
$$

where $L$ is a constant depending on and $f$ and $d(X, E)$ is the distance between $X$ and $E$ defined as:

$$
d(X, E)=\inf \{|t-x|: t \in E\} .
$$

Proof. Let $\bar{E}$ denote the closure of $E$ in $[0,+\infty)$. Then, there exist a point $x_{0} \in \bar{E}$ such that $d\left(x_{0}, E\right)=\left|x-x_{0}\right|$. Using the triangle inequality

$$
|f(t)-f(x)| \leq\left|f(t)-f\left(x_{0}\right)\right|+\left|f(x)-f\left(x_{0}\right)\right|,
$$

by monotonicity of operators $T_{n, p}^{\alpha, \beta}$ and (4.1) we get:

$$
\begin{gathered}
\left|T_{n, p}^{(\alpha, \beta)}(f, q, x)-f(x)\right| \leq T_{n, p}^{(\alpha, \beta)}\left(\left|f(t)-f\left(x_{0}\right)\right|, q, x\right)+T_{n, p}^{(\alpha, \beta)}\left(\left|f(x)-f\left(x_{0}\right)\right|, q, x\right) \leq \\
\leq L\left\{T_{n, p}^{(\alpha, \beta)}\left(\left|t-x_{0}\right|^{a}, q, x\right)+\left|x-x_{0}\right|^{a}\right\} \leq L\left\{T_{n, p}^{(\alpha, \beta)}\left(|t-x|^{a}+\left|x-x_{0}\right|^{a}, x\right)+\left|x-x_{0}\right|^{a}\right\}= \\
=L\left\{T_{n, p}^{(\alpha, \beta)}\left(|t-x|^{a}, q, x\right)+2\left|x-x_{0}\right|^{a}\right\} .
\end{gathered}
$$

Using Hölder's inequality with $\frac{1}{p_{1}}=\frac{a}{2}, \quad \frac{1}{q_{1}}=1-\frac{1}{p_{1}}$, we get,

$$
\begin{gathered}
\left|T_{n, p}^{(\alpha, \beta)}(f, q, x)-f(x)\right| \leq L\left\{\left[T_{n, p}^{(\alpha, \beta)}\left(|t-x|^{a p_{1}}, q, x\right)\right]^{\frac{1}{p_{1}}}\left[T_{n, p}^{(\alpha, \beta)}\left(1^{q_{1}}, q, x\right)\right]^{\frac{1}{q_{1}}}+2 d(X, E)^{a}\right\} \\
=L\left\{\left[T_{n, p}^{(\alpha, \beta)}\left(|t-x|^{2}, q, x\right)\right]^{\frac{\alpha}{2}}+2(d(X, E))^{a}\right\},
\end{gathered}
$$


the desired result mediately.

\section{Local approximation}

Let $C_{B}[0,+\infty)$ be the space of all real-valued continuous and bounded functions $f$ on $[0,+\infty)$, endowed with the norm $\|f\|=\sup _{x \in[0,+\infty)}|f(x)|$.

The Petree's $K$ - functional is defined by:

$$
K_{2}(f ; \delta)=\inf _{g \in C_{[0,+\infty)}^{2}}\left\{\|f-g\|+\delta\left\|g^{\prime \prime}\right\|\right\}
$$

where $C_{B}^{2}[0,+\infty)=\left\{g \in C_{B}[0,+\infty): g^{\prime}, g^{\prime \prime} \in C_{B}[0,+\infty)\right\}$.

By [3] there exists an absolute constant $M>0$ such that

$$
K_{2}(f, \delta) \leq M \omega_{2}(f ; \sqrt{\delta}),
$$

where $\delta>0$ and the second-order modulus of smoothness is defined as:

where $f \in C_{B}[0,+\infty)$ and $\delta>0$. Also, we let

$$
\omega_{2}(f ; \sqrt{\delta})=\sup _{0<h \leq \delta} \sup _{x \in[0,+\infty)}|f(x+2 h)-2 f(x+h)+f(x)|,
$$

$$
\omega_{2}(f ; \delta)=\sup _{0<h \leq \delta} \sup _{x \in[0,+\infty)}|f(x+h)-f(x)| .
$$

Lemma 5.1. For $f \in C_{B}[0,+\infty)$, we have

$$
\left\|T_{n, p}^{(\alpha, \beta)} f\right\| \leq\|f\| .
$$

Proof. Since $p_{n+p, k}(q ; x) \geq 0$ for all $0<q<1$ and $x \in\left[0, b_{n}\right]$, then from (1) and Lemma 2.1 we get the following:

$$
\left|T_{n, p}^{(\alpha, \beta)}(f ; q, x)\right| \leq \sum_{k=0}^{n+p} p_{n+p, k}(q ; x) \int_{0}^{1} \mid f\left(\frac{t b_{n}}{[n+1+\beta]_{q}}+\frac{q[k+\alpha]_{q} b_{n}}{[n+1+\beta]_{q}} \mid d_{q} t \leq\|f\| T_{n, p}^{(\alpha, \beta)}(1, q, x)=\|f\| .\right.
$$

Theorem 5.1. For all $f \in C_{B}[0,+\infty), x \in\left(0, b_{n}\right], q \in(0,1)$ and $p \in N$ fixed, there exist $c>0$ such that

$$
\left|T_{n, p}^{(\alpha, \beta)}(f ; q, x)-f(x)\right| \leq c \omega_{2}\left(f, \sqrt{\lambda_{n, q}(x)}\right)+\omega\left(f, \mu_{n, q}(x)\right)
$$

where $\lambda_{n, q}=2\left(\frac{q^{\alpha+1}[n+p]_{q}}{[n+1+\beta]_{q}}-1\right)^{2} x^{2}+4 \frac{[n+p]_{q} b_{n}(1+\alpha)}{[n+1+\beta]_{q}+q^{2}} q^{\alpha+1}+\frac{2 b_{n}^{2}}{[n+1+\beta]_{q}}(1+\alpha)^{2}$, and $\mu_{n, q}=\left(\frac{q^{\alpha+1}[n+p]_{q}}{[n+1+\beta]_{q}}-1\right) x+$ $\frac{b_{n}}{[n+1+\beta]_{q}}(\alpha+1)$.

Proof. For $f \in C_{B}[0,+\infty)$ we define operator $T_{n, p}^{*(\alpha, \beta)}(f ; q, x): C_{B}[0,+\infty) \rightarrow C_{B}[0,+\infty)$ by

$$
T_{n, p}^{*(\alpha, \beta)}(f ; q, x)=T_{n, p}^{(\alpha, \beta)}(f ; q, x)+f(x)-f\left(u_{n} x+v_{n}\right)
$$

where $u_{n}=\frac{q^{\alpha+1}[n+p]_{q}}{[n+1+\beta]_{q}} x$ and $v_{n}=\frac{b_{n}}{[n+1+\beta]_{q}}\left(\frac{1}{[2]_{q}}+[\alpha]_{q} q\right)$.

Then, by Lemma 2.1, we have $T_{n, p}^{*(\alpha, \beta)}((t-x) ; q, x)=0$.

For given $g \in C_{B}^{2}[0,+\infty)$, using Taylor's formula

we obtain:

$$
g(y)-g(x)=(y-x) g^{\prime}(x)+\int_{x}^{y}(y-u) g^{\prime \prime}(u) d u
$$

$$
\begin{aligned}
& T_{n, p}^{*(\alpha, \beta)}(g ; q, x)=g(x)+T_{n, p}^{*(\alpha, \beta)}\left(\left(\int_{x}^{y}(y-u) g^{\prime \prime}(u) d u\right) ; q, x\right)= \\
& =g(x)+T_{n, p}^{(\alpha, \beta)}\left(\int_{x}^{y}(y-u) g^{\prime \prime}(u) d u ; q, x\right)-\int_{x}^{u_{n} x+v_{n}}\left(u_{n} x+v_{n}-u\right) g^{\prime \prime}(u) d u .
\end{aligned}
$$

Since,

$$
\left|\int_{x}^{y}(y-u) g^{\prime \prime}(u) d u\right| \leq \int_{x}^{y}|(y-u)| \cdot\left|g^{\prime \prime}(u)\right| d u \leq\left\|g^{\prime \prime}\right\| \int_{x}^{y}|y-u| d u \leq\left\|g^{\prime \prime}\right\|(y-x)^{2},
$$


and

$$
\int_{x}^{u_{n} x+v_{n}}\left(u_{n} x+v_{n}-u\right) g^{\prime \prime}(u) d u \leq\left\|g^{\prime \prime}\right\|\left\{\left(u_{n}-1\right) x+v_{n}\right\}^{2}
$$

we get

$$
\begin{gathered}
\left|T_{n, p}^{*(\alpha, \beta)}(g ; q, x)-g(x)\right|=\left|T_{n, p}^{(\alpha, \beta)}\left(\int_{x}^{y}(y-u) g^{\prime \prime}(u) d u ; q, x\right)-\int_{x}^{u_{n} x+v_{n}}\left(u_{n} x+v_{n}-u\right) g^{\prime \prime}(u) d u\right| \leq \\
\leq\left\|g^{\prime \prime}\right\|\left\{T_{n, p}^{(\alpha, \beta)}\left((y-x)^{2} ; q ; x\right)+\left\{\left(u_{n}-1\right) x+v_{n}\right\}^{2}\right\} .
\end{gathered}
$$

Hence Lemma 2.1 implies that

$$
\begin{aligned}
\left|T_{n, p}^{(\alpha, \beta)}(g ; q, x)-g(x)\right| \leq\left\|g^{\prime \prime}\right\| & \left\{\left(\frac{q^{2 \alpha+3}[n+p]_{q}[n+p-1]_{q}}{[n+1+\beta]_{q}^{2}}-2 \frac{q^{\alpha+1}[n+p]_{q}}{[n+1+\beta]_{q}}+1\right) x^{2}+\right. \\
+ & {\left[\frac{[n+p]_{q}}{[n+1+\beta]_{q}^{2}} b_{n}\left(\frac{2}{[2]_{q}} q^{\alpha+1}+q^{\alpha+2}\left(2[\alpha]_{q}+q^{\alpha}\right)\right)-2 \frac{b_{n}}{[n+1+\beta]_{q}}\left(\frac{1}{[2]_{q}}+q[\alpha]_{q}\right)\right] x+} \\
& \frac{b_{n}^{2}}{[n+1+\beta]_{q}^{2}}\left(\frac{1}{[3]_{q}}+\frac{2 q[\alpha]_{q}}{[2]_{q}}+q^{2}[\alpha]_{q}^{2}\right)+\left[\left(u_{n}-1\right) x+v_{n}\right] .
\end{aligned}
$$

Thus, $\left\|T_{n, p}^{*(\alpha, \beta)}(f, q, x)\right\| \leq 3\|f\|$ for all $f \in C_{B}[0, \infty)$.

Now, for $f \in C_{B}[0,+\infty)$ and $g \in C_{B}^{2}[0, \infty)$, we obtain:

$$
\begin{aligned}
\left|T_{n, p}^{*(\alpha, \beta)}(f, q, x)-f(x)\right| & =\left|T_{n, p}^{*(\alpha, \beta)}(f, q, x)-f(x)+f\left(u_{n} x+v_{n}\right)\right| \\
\leq & \left|T_{n, p}^{*(\alpha, \beta)}(f-g, q, x)-g(x)\right|+\left|T_{n, p}^{*(\alpha, \beta)}(g, q, x)-g(x)\right|+|g(x)-f(x)| \\
& +\left|f\left(u_{n} x+v_{n}\right)-f(x)\right| \leq 4\|f-g\|+\lambda_{n, q}(x)\left\|g^{\prime \prime}\right\|+\omega\left(f, \mu_{n, q}(x)\right) .
\end{aligned}
$$

Now, taking infimum on the right side over all $g \in C_{B}^{2}[0,+\infty)$, we get the following result :

where:

$$
\left|T_{n, p}^{(\alpha, \beta)}(f, q, x)-f(x)\right| \leq 4 K\left(f ; \lambda_{n, q}(x)\right)+\omega\left(f, \mu_{n, q}(x)\right) \leq c \omega\left(f ; \sqrt{\lambda_{n, q}(x)}\right)+\omega\left(f ; \mu_{n, q}(x)\right),
$$

$$
\begin{aligned}
& \lambda_{n, q}(x)=2\left(\frac{q^{\alpha+1}[n+p]_{q}}{[n+1+\beta]_{q}}-1\right)^{2} x^{2}+4 \frac{[n+p]_{q} b_{n}(1+\alpha)}{[n+1+\beta]_{q}+q^{2}} q^{\alpha+1}+\frac{2 b_{n}^{2}}{[n+1+\beta]_{q}}(1+\alpha)^{2}, \\
& \mu_{n, q}(x)=\left(\frac{q^{\alpha+1}[n+p]_{q}}{[n+1+\beta]_{q}}-1\right) x+\frac{b_{n}}{[n+1+\beta]_{q}}(\alpha+1) .
\end{aligned}
$$

Example 1. For $n=100$, and $500, \alpha=0, \beta=5, p=5, q=\frac{n}{n+1}$ and $b_{n}=\ln n$, the convergence of $T_{n, p}^{(\alpha, \beta)}$ to $f(x)=$ $x^{2}$ is illustrated in Figure (a) respectively Figure (b).

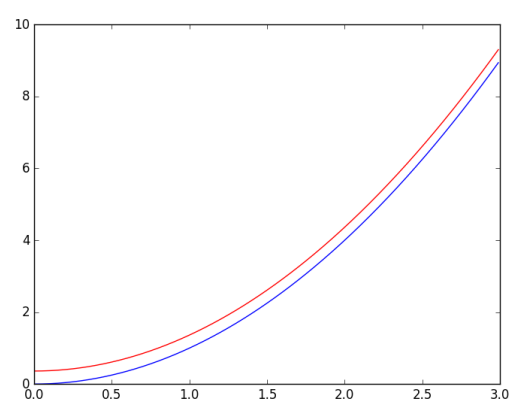

(a)

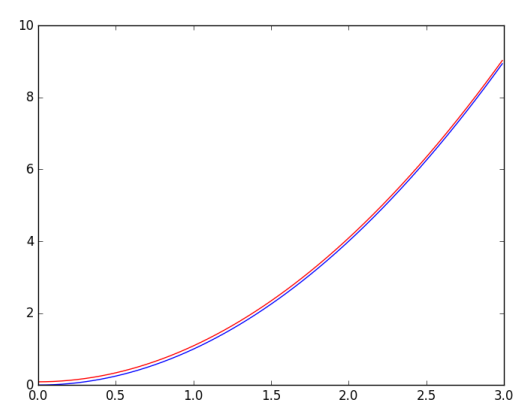

(b) 


\section{Acknowledgment}

The authors are extremely thankful to the reviewers for critical reading of the manuscript leading to better presentation of the paper.

\section{References}

[1] Agratini, O., and Ogün D.. "Weighted approximation by q-Szász-King type operators." Taiwanese Journal of Mathematics (2010): 1283-1296.

[2] Agrawal, P. N., Vijay Gupta, and A. Sathish Kumar. "On q-analogue of Bernstein-Schurer-Stancu operators." Applied Mathematics and Computation 219.14 (2013): 7754-7764.

[3] Aral, A., V. Gupta, and R. P. Agarwal. Applications of q-calculus in operator theory. New York: Springer, 2013.

[4] Area, I., et al. "Formulae relating little q-Jacobi, q-Hahn and q-Bernstein polynomials: application to q-Bézier curve evaluation." Integral Transforms and Special Functions 15.5 (2004): 375-385.

[5] Dalmanoglu, Özge. "Approximation by Kantorovich type q-Bernstein operators." Proceedings of the 12th WSEAS International Conference on Applied Mathematics. World Scientific and Engineering Academy and Society (WSEAS), 2007.

[6] Duman, O., and C. Orhan. "Statistical approximation by positive linear operators." Studia Mathematica 161.2 (2004): 187-197.

[7] Freedman, Allen, and John Sember. "Densities and summability." Pacific Journal of Mathematics 95.2 (1981): 293-305.

[8] Gadjiev, A. D., and C. Orhan. "Some approximation theorems via statistical convergence." Rocky Mountain J. Math 32.1 (2002).

[9] Gal, S. G., and V. Gupta. "Approximation of vector-valued functions by q-Durrmeyer operators with applications to random and fuzzy approximation." Oradea Univ. Math. J 16 (2009): 233-242.

[10] Gupta, Vijay. "Some approximation properties of q-Durrmeyer operators." Applied Mathematics and Computation 197.1 (2008): 172-178.

[11] Gupta, Vijay, and Cristina Radu. "Statistical approximation properties of q-Baskakov-Kantorovich operators." Open Mathematics 7.4 (2009): 809-818.

[12] Kac, V., and Pokman Ch.. Quantum calculus. Springer Science \& Business Media, 2001.

[13] Karsli, H., and Vijay G.. "Some approximation properties of q-Chlodowsky operators." Applied Mathematics and Computation 195.1 (2008): 220-229.

[14] Lin, Qiu. "Statistical Approximation of-Bernstein-Schurer-Stancu-Kantorovich Operators." Journal of Applied Mathematics 2014 (2014).

[15] Lupas, A. A q-analogue of the Bernstein operator. University of Cluj-Napoca, Seminar on numerical and statistical calculus, Nr. 9, (1987).

[16] Ostrovska, S. "The sharpness of convergence results for q-Bernstein polynomials in the case q> 1." Czechoslovak Mathematical Journal 58.4 (2008): 1195-1206.

[17] Ostrovska, Sofiya. "On the image of the limit q-Bernstein operator." Mathematical Methods in the Applied Sciences 32.15 (2009): 1964-1970.

[18] Phillips, George M. "Bernstein polynomials based on the q-integers." Annals of Numerical Mathematics 4 (1996): 511-518.

[19] Ren, Mei-Ying, and Xiao-Ming Zeng. "On statistical approximation properties of modified q-Bernstein-Schurer operators." Bulletin of the Korean Mathematical Society 50.4 (2013): 1145-1156.

[20] Vedi, T., and Mehmet A. Ö. "Chlodowsky-type q-Bernstein-Stancu-Kantorovich operators." Journal of Inequalities and Applications 2015.1 (2015): 91. 


\section{Affiliations}

BEHAR BAXHAKU

AdDRESS: Department of Mathematics, University of Prishtina, Mother Teresa, 10000 Prishtina, Kosova. E-MAIL: behar.baxhaku@uni-pr.edu

FEVZI BERISHA

AdDRESS: Department of Mathematics, University of Prishtina, Bregu i Diellit 10000 Prishtina, Kosova. E-MAIL: fevzi.berisha@uni-pr.edu 